\title{
Mesure de l'activité des saponines de la luzerne par les larves du ver de farine: Tenebrio molitor L. (Coléoptère, Tenebrionidae). I. - Comparaison avec les résultats de divers tests biologiques
}

\author{
Pascale PRACROS \\ avec la collaboration technique de Christine Couranjou \\ I.N.R.A., Station de Zoologie, Centre de Recherches de Bordeaux, F 33140 Pont-de-la-Maye
}

Devant les difficultés du dosage chimique de la teneur en saponines des variétés de luzerne, on a recours à différents tests biologiques dits " de nuisance " fondés sur la propriété de séquestration du cholestérol de certaines molécules existant dans le mélange de saponines. Cette propriété est responsable de l'inhibition de croissance d'un champignon (Trichoderma viride) et de la lyse membranaire des hématies. La méthode de dosage biologique de référence est effectuée sur des poussins, animaux chez lesquels l'ingestion de saponines provoque une chute de croissance et une baisse des quantités d'aliments consommés.

L'estimation de la qualité nutritionnelle de divers aliments protéiques pour la larve de Tenebrio molitor a montré la grande sensibilité de cet insecte aux substances allélochimiques des plantes. La comparaison des performances de ces larves (quantité d'aliment assimilé et croissance pondérale) nourries à partir de diverses variétés de luzerne aux dosages biologiques des saponines par les tests Trichoderma, hémolyse et poussin montre que cet insecte est très sensible à la présence de saponines dans son aliment. Une addition de cholestérol dans le régime alimentaire est à l'origine d'une amélioration très hautement significative des performances des larves, ce qui confirme que l'activité antinutritionnelle observée est bien imputable aux saponines.

Si le test Tenebrio ne permet pas le dosage précis des saponines de la luzerne, les résultats des différents essais biologiques sont suffisamment corrélés entre eux pour permettre d'envisager l'utilisation de ce nouveau test dans les programmes d'amélioration des variétés de luzerne.

Mots clés additionnels : Substances allélochimiques, Trichoderma viride, hémolyse, poussin.

Measure of biological activity of lucerne saponins by yellow mealworm larvae, Tenebrio molitor $L$. I. - Comparison with results of some biological tests.

Biological tests are required to determine the saponin content of lucerne because of the difficulty in determining these substances chemically. A new method of assessing the biological effect of lucerne saponins, the "Tenebrio molitor test », was applied to different samples of lucerne. The results were compared with the biological assay of saponins by the traditional biological tests : growth inhibition of Trichoderma viride, the hemolysis test and variation of consumption and growth by chicks. All these methods classified lucerne samples consistently (correlation coefficient $\mathrm{r}=0.97$ between Tenebrio and Trichoderma and between Tenebrio and hemolysis in the first assay and correlation coefficient $\mathrm{r}=0.65$ between Tenebrio and Trichoderma, 0.90 between Tenebrio and hemolysis and 0.87 between Tenebrio and chicks in the second assay). The improvement in the nutritional value of lucerne for Tenebrio larvae obtained by incorporation of cholesterol shows the antinutritional activity of saponins. These results showed that the Tenebrio test could be used for the biological assay of lucerne saponins, in support of plant breeding programs.

Additional key words : Allelochemical compounds, Trichoderma viride, hemolysis, chick growth. 


\section{INTRODUCTION}

La luzerne, Medicago sativa L., est une source protéique très productive des régions à climat tempéré. $\mathrm{A}$ titre de comparaison, un hectare de luzerne produit 2 tonnes de protéine par an tandis qu'un hectare de soja en fournit environ 1 tonne. Mais les variétés de luzerne cultivées contiennnent des taux élevés de saponines, hétérosides composés de sapogénines (triterpènes) et de sucres (GESTETNER, 1971). Certains de ces composés présentent une activité antinutritionnelle attribuable à plusieurs causes dont l'importance varie avec l'espèce " consommatrice ": effet dissuadant de la prise de nourriture dû à l'amertume, chez le poussin (CHEEKE, 1976), troubles physiologiques chez les mammifères, les oiseaux et les animaux à sang froid liés à la séquestration du cholestérol par l'acide médicagénique (ISHAYAA et al., 1969) et à l'inhibition d'enzymes dont par exemple l' $\alpha$-chymotrypsine et la cholinestérase (CHEEKE, 1971 ; APPLEBAUM \& BIRK, 1979).

Pour étudier les propriétés antinutritionnelles des saponines de la luzerne, l'expérience de référence est réalisée sur poulets, animaux particulièrement sensibles à ces substances et pour lesquels s'est engagé un important programme d'amélioration de la qualité nutritionnelle de cette plante (actuellement les protéines de luzerne n'entrent dans la ration des volailles que pour 2,5 p. 100). Ce type d'expérimentation nécessite de grandes quantités de matières premières puisqu'il s'agit de nourrir une dizaine de poulets pendant 1 à 4 semaines afin de contrôler leur appétit et leur croissance. Ces essais sont coûteux et ne peuvent être appliqués au grand nombre d'échantillons dont dispose chaque année le sélectionneur. Pour estimer l'activité antinutritionnelle attribuable aux saponines, on a recours à d'autres tests biologiques qui nécessitent de faibles quantités de matières premières : les tests Trichoderma et hémolyse. L'activité hémolytique serait liée à la formation de complexes indissociables entre les saponines à sapogénines acides et le cholestérol de la membrane cellulaire (SHANY et $a l ., 1980$ ) et un mécanisme physiologique du même ordre serait à l'origine de l'inhibition de croissance du champignon Trichoderma viride Pers. (LEATH et al., 1972). Les travaux de PEDERSEN et WANG (1971) ont montré la corrélation existant entre les teneurs en saponines de diverses variétés de luzerne et leur activité sur la croissance de $T$. viride et ont permis d'établir une première échelle de référence entre les résultats biologiques et les teneurs en ces substances.

Le mode d'action des saponines sur les insectes n'est pas encore entièrement élucidé. Un doute subsiste sur les rôles respectifs de la dissuasion alimentaire et de l'antibiose (HORBER et al., 1974). Chez Locusta migratoria $\mathrm{L}$., la réduction de croissance serait due à la fois à des troubles du mécanisme de réabsorption de l'eau par fixation des saponines sur les membranes des cellules intestinales et à l'inhibition de l'activité d'enzymes (APPLEBAUM \& BIRK, 1979 ; BIRK \& PERI, 1980). Des saponines extraites de racines de luzerne (GESTETNER et al., 1972) ou leurs sapogénines acides (SHANY et al., 1980) incorporées au régime alimentaire des larves de Tribolium castaneum (Herbst.) entraînent une réduction de croissance d'autant plus faible que le taux de cholestérol ajouté à la ration augmente. La propriété de séquestration du cholestérol par les saponines a un effet très important chez l'insecte qui est incapable de synthétiser le noyau stéroïde indispensable à l'édification des membranes cellulaires et à la production de l'ecdysone. Une carence en stérols provoque une baisse de croissance, un ralentissement du développement et une diminution de la reproduction (RITTER \& WIENTJENS, 1967).

Nous avons déjà mis en évidence les caractéristiques antinutritionnelles de certaines variétés de luzerne pour les larves du ver de farine, Tenebrio molitor L. (PRACROS, 1984), propriétés probablement liées à la présence de saponines. Parmi un grand nombre d'essais entrepris depuis 1982 dans l'activité du groupe de travail "saponines/luzerne ", nous avons choisi de présenter les résultats les plus représentatifs de ceux obtenus dans divers laboratoires et faisant intervenir plusieurs méthodes d'estimation des teneurs en saponines de la luzerne.

\section{MATÉRIEL ET MÉTHODE}

Les échantillons étudiés (poudre de plante entière et poudre de feuille) ont été fournis par la station d'amélioration des plantes de Lusignan (I.N.R.A.) aux différents partenaires du groupe de travail. Ils ont été prélevés et conditionnés à Lusignan à partir de cultures menées sur les terrains de cette station. Nous présentons ici les résultats obtenus à partir de poudre de plantes entières de 10 variétés de luzerne et de poudre de feuilles de 4 variétés plus ou moins riches en saponines. Quant aux échantillons de concentrés protéiques de luzerne (PX) étudiés, ils ont été obtenus industriellement par la société France-Luzerne à partir de la culture de 8 variétés réalisée en 1982 dans l'Aube.

Les aliments expérimentaux sont préparés de telle sorte qu'ils correspondent à l'optimum de consommation et de croissance des insectes. Les quantités de matière première à introduire dans les régimes ont été définies par des essais " doses " préalables $(20$ p. 100 de poudre de plante entière, 10 p. 100 de poudre de feuilles et 7 p. 100 de concentré protéique).

Les farines de luzerne sont complémentées par de grandes quantités de glucose $(80 \mathrm{p} .100$ dans les plantes entières, 90 p. 100 dans les feuilles et 93 p. 100 dans les concentrés protéiques), par un mélange de sels minéraux selon la formulation de Mac Collum adaptée aux besoins des insectes $(4 \mathrm{~g}$ dans $100 \mathrm{~g}$ du mélange précédemment décrit), en vitamines du groupe B, en carnitine et en chlorure de zinc reconnus indispensables aux besoins de T. molitor (FRAENKEL et al., 1950). Les régimes expérimentaux sont volontairement privés de cholestérol pour mettre en évidence de façon plus nette les éventuelles différences de teneur en saponines des variétés de luzerne. Un essai particulier, effectué à partir d'échantillons de feuilles, a été réalisé avec incorporation de quantités croissantes de cholestérol $(0 ; 0,5$; $1 ; 1,5$ et 2 p. 100$)$.

L'expérience avec les larves de Tenebrio molitor consiste à nourrir les jeunes larves de cet insecte avec les aliments dont on désire obtenir un classement en fonction de leur qualité nutritionnelle pour l'insecte, qualité estimée par la quantité d'aliment sec métabolisé 
(ASM en mg par larve = quantité d'aliment sec fourni aux larves en début d'essai - quantité d'aliment sec non consommé - quantité de fèces produites par les insectes pendant l'essai), le gain de poids sec des insectes (GPS en mg par larve) et l'indice de conversion de l'aliment métabolisé en biomasse (IS = ASM/GPS).

Cinq lots de 20 larves âgées de 7 semaines et pesant chacune environ $15 \mathrm{mg}$ sont placés pendant 4 semaines sur chacun des aliments dans une enceinte climatisée $\left(25^{\circ} \mathrm{C}\right.$ et $80 \mathrm{p} .100$ d'humidité relative).

En début d'expérimentation, un échantillon de chaque aliment préparé et un lot de larves semblables à celles qui sont utilisées pour le test sont desséchés sous vide pendant $24 \mathrm{~h}$ à $50^{\circ} \mathrm{C}$ afin de déterminer leur teneur en matière sèche. En fin d'essai, régimes non consommés et larves sont, après pesée, traités de la même façon.

Ces mêmes échantillons de luzerne ont subi les essais biologiques permettant d'estimer leurs teneurs en saponines: essais réalisés sur le champignon $T$. viride dont la taille des colonies est inversement proportionnelle au taux de saponines présent dans le milieu de culture (W. PIETRASZEK en Italie), sur les hématies dont la membrane fixe les saponines ce qui provoque une hémolyse que l'on peut mesurer par colorimétrie (I. BOCSA en Hongrie), et sur de jeunes poussins dont les performances zootechniques de croissance et de consommation sont perturbées par l'ingestion des saponines de la luzerne (L. LACASSAGNE à l'I.N.R.A. de Tours).

\section{RÉSULTATS}

\section{A. Les performances de l'insecte}

L'insecte accepte les régimes alimentaires à base de luzerne et, même dans les cas les plus défavorables, aucune des variétés n'a été refusée. Le régime préparé avec la poudre de feuille de la variété Lahontan $(10 \mathrm{~g})$ et du cholestérol $(2 \mathrm{~g})$ donne des résultats de consommation (119 mg par larve) (tabl. 3) identiques à ceux des insectes élevés dans les mêmes conditions expérimentales sur de la farine intégrale de blé tendre qui est leur aliment habituel. La croissance pondérale des larves nourries avec ce régime "luzerne », $16 \mathrm{mg}$ par larve, reste toutefois inférieure à celle obtenue sur l'aliment témoin, $28 \mathrm{mg}$ par larve. Le régime expérimental à base de luzerne, quoique bien assimilé par cet insecte, n'est pas aussi bien converti en biomasse que le blé (1 $\mathrm{mg}$ d'insecte est " produit » soit par l'assimilation de $4,3 \mathrm{mg}$ de farine de blé soit par celle de 7,4 $\mathrm{mg}$ de régime luzerne).

La régression linéaire des gains de poids secs des larves sur les quantités d'aliments secs assimilés (fig. 1) est très hautement significative avec un coefficient de corrélation entre ces 2 variables de 0,96 avec les poudres de plantes entières, 0,98 avec les concentrats protéiques et 0,99 avec les poudres de feuilles pour respectivement 10,8 et 20 couples de données.

Pour une quantité d'aliment sec assimilé, la croissance des insectes est plus importante lorsque le régime contient le concentrat protéique et l'aliment le moins bien converti est celui qui est préparé à base de poudres de plantes entières. Ce résultat ne trouve pas d'explica-

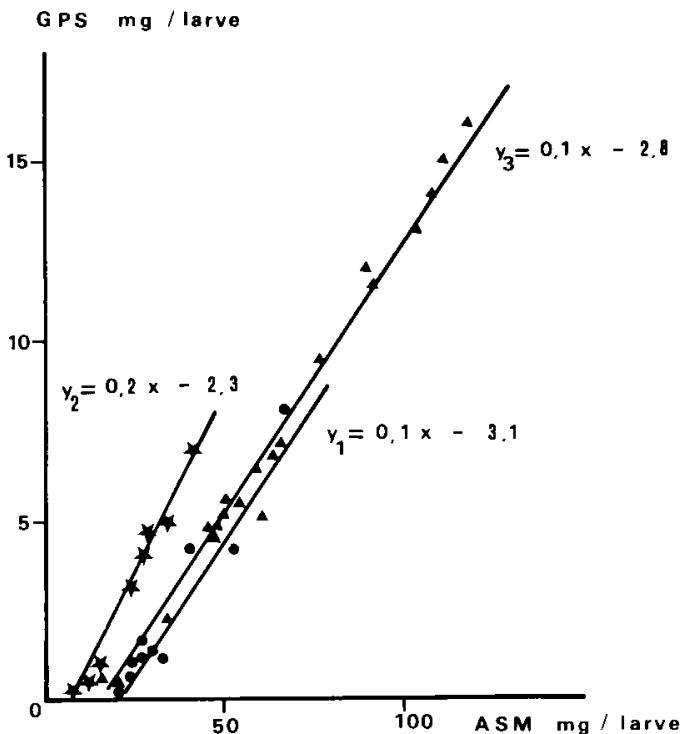

Figure 1

Droites de régression du gain de poids sec des larves sur la quantité d'aliment sec métabolisé. Les résultats du premier essai sont représentés par $\bullet$, ceux du second par $\star$ et ceux du troisième par $\boldsymbol{\Lambda}$.

Regression line of dry weight gain of insects on the food assimilated. Results of the first experiment are showed by $\bullet$, the second by $\star$ and the third by $\mathbf{\Lambda}$

tion quand on considère la teneur en matière azotée des différents régimes expérimentaux puisque l'on passe de 3 p. 100 de matière azotée totale avec les concentrats, à 2,7 p. 100 avec les feuilles et à 3,2 p. 100 avec les plantes entières. Mais la nature de ces protéines ou encore la teneur en cellulose brute de ces divers échantillons étudiés $(0,2$ p. 100 dans les régimes à base de concentrats protéiques, 2 p. 100 dans les régimes à base de feuilles et 5 p. 100 dans ceux contenant les plantes entières) peuvent être des éléments d'explication des différences de qualité nutritionnelle observées.

\section{B. Le classement des variétés}

Les analyses de variance appliquées à ces données (tabl. 1 et 2) ont permis de classer les sources de luzerne en fonction de leur qualité nutritionnelle pour T. molitor. Nous pouvons remarquer que, quelle que soit l'origine de l'échantillon de luzerne (année et lieu de culture, récolte manuelle et conditionnement à l'échelle du laboratoire ou industriellement) et quel que soit l'organe étudié (plante entière, feuille), le classement des variétés reste le même : Lahontan, Robot et Ruro ont une valeur nutritionnelle supérieure aux variétés Europe, Orca et Lutèce.

\section{La comparaison avec les autres tests}

Les teneurs en saponines des échantillons étudiés ayant été estimées par les tests biologiques « Trichoderma » et " hémolyse " et, pour certains d'entre eux, par le test de référence sur poussins, nous avons comparé les résultats obtenus par l'application de ces différentes méthodes par calcul des coefficients de corréla- 
TABLEAU 1

Valeur nutritionnelle de la plante entière de 10 variétés de luzerne pour la larve de Tenebrio molitor.

Les résultats sont donnés sous la forme de la moyenne de 5 répétitions affectée de l'intervalle de confiance à $95 \%$. Deux moyennes suivies de la même lettre ne sont pas significativement différentes au seuil de $5 \%$ (test de Duncan).

Nutritional value of 10 varieties of lucerne for the yellow mealworm larvae Tenebrio molitor.

Results are the average of 5 replicates. The mean value is given with confidence limits at the $95 \%$ confidence level. Two means followed by the same letter are not significantly different at the $5 \%$ level (Duncan test).

\begin{tabular}{|c|c|c|c|c|}
\hline $\begin{array}{l}\text { VARIÉTÉS } \\
\text { DE LUZERNE }\end{array}$ & $\begin{array}{c}\mathrm{ASM} \\
\mathrm{mg} / \text { larve }\end{array}$ & $\begin{array}{c}\text { GPS } \\
\mathrm{mg} / \text { larve }\end{array}$ & $\begin{array}{l}\text { TAUX DE } \\
\text { mg/100 g de régim } \\
\text { TRICHODERMA }\end{array}$ & $\begin{array}{l}\text { ONINES } \\
\text { imés par les tests } \\
\text { HÉMOLYSE }\end{array}$ \\
\hline LAHONTAN & $66,1(4,2) \mathrm{a}$ & $8,1(0,5) \mathbf{a}$ & 6,0 & 6,0 \\
\hline ROBOT & $52,8(3,1) \mathrm{b}$ & $4,2(0,9) \mathrm{b}$ & 10,4 & 10,0 \\
\hline RURO & $40,2(1,5) c$ & $4,2(0,4) \mathrm{b}$ & 14,0 & 11,8 \\
\hline RESIS & $32,8(1,2) \mathrm{d}$ & $1,2(1,0) \mathrm{c}$ & 19,2 & 12,6 \\
\hline MAGALI & $29,8(0,8) \mathrm{e}$ & $1,4(0,4) \mathrm{c}$ & 18,4 & 12,0 \\
\hline EUROPE & $26,8(0,8) \mathrm{f}$ & $1,2(0,5) \mathrm{c}$ & 19,9 & 13,0 \\
\hline MARIS-KABUL & $25,4(0,7) \mathrm{fg}$ & $1,4(0,4) \mathrm{c}$ & 17,7 & 13,4 \\
\hline LUTECE & $24,0(0,7) \mathrm{g}$ & $1,2(0,3) \mathrm{c}$ & 19,4 & 13,2 \\
\hline FLAMANDE & $24,0(0,2) \mathrm{g}$ & $0,6(0,2) \mathrm{d}$ & 20,9 & $i \tilde{3}, 8$ \\
\hline ORCA & $21,8(1,1) \mathrm{h}$ & $0,2(0,5) d$ & 19,0 & 14,2 \\
\hline
\end{tabular}

ANALYSE DE VARIANCE

Variable étudiée : ASM

$\mathrm{F}=403$ avec $9 \mathrm{ddl}$ l'effet « variété » est très hautement significatif ( $\mathrm{F}$ table $=2,12$ au seuil de $1 \%$ \% $)$.

Variable étudiée : GPS

$\mathrm{F}=264$ avec 9 ddl l'effet " variété » est très hautement significatif ( $\mathrm{F}$ table $=2,12$ au seuil de $1 \%$ oo).

\section{COEFFICIENTS DE CORRÉLATION ENTRE LES RÉSULTATS DES TESTS BIOLOGIQUES}

TENEBRIO ASM

TENEBRIO GPS

TRICHODERMA

HÉMOLYSE

TENEBRIO ASM

TENEBRIO GPS

TRICHODERMA

HÉMOLYSE

\section{1}

$0,96^{* * *}$

$-0,97^{* * *}$

$-0,97 * * *$
1

$-0,97^{* * *}$

$-0,96 * * * \quad 0,94 * * *$
1 tion (tabl. 3 et fig. 2 et 3 ). Les variétés de luzerne pauvres en saponines d'après les tests Trichoderma et hémolyse (Lahontan, Robot et Ruro) se caractérisent par une efficacité nutritionnelle supérieure chez l'insecte. Ces résultats confirment l'effet antinutrition-

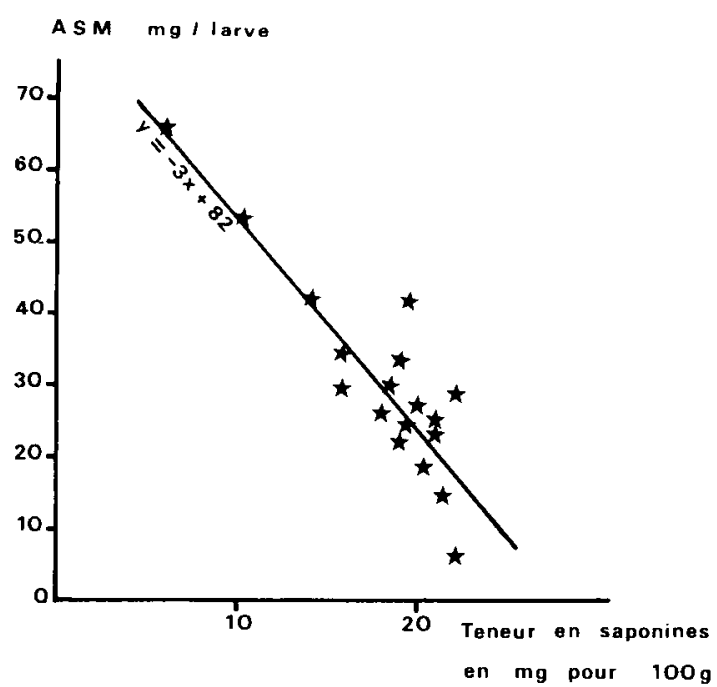

Figure 2

Droite de regression de la quantité d'aliment sec métabolisé par Tenebrio molitor sur la teneur en saponines estimée par le test Trichoderma.

Regression line of dry food assimilated by Tenebrio molitor on the saponin level estimated by the Trichoderma test.

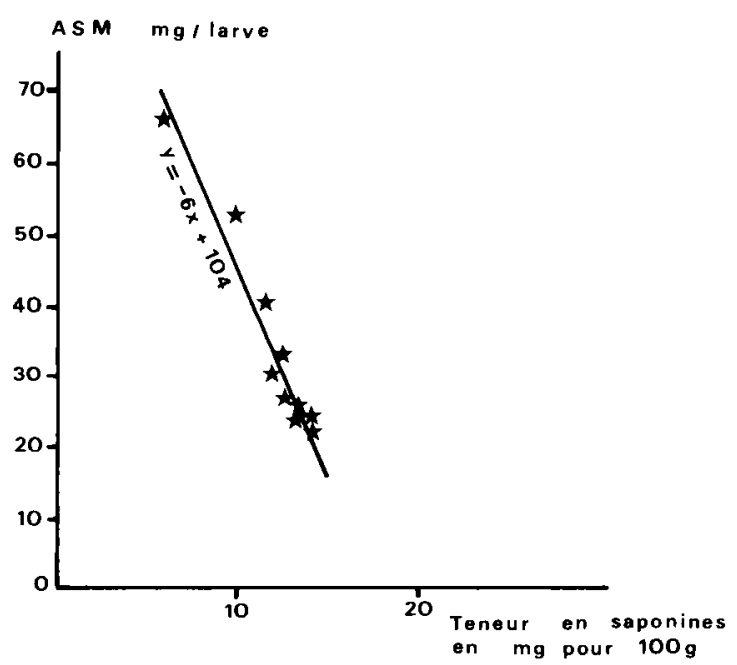

Figure 3

Droite de régression de la quantité d'aliment sec métabolisé par Tenebrio molitor sur la teneur en saponines estimée par le test Hémolyse.

Regression line of dry food assimilated by Tenebrio molitor on the saponin level estimated by the hemolysis test.

nel dominant de ces substances. Les tests Tenebrio et Hémolyse sont corrélés entre eux de façon très hautement significative dans les 2 essais entrepris tandis que les résultats du test Trichoderma paraissent plus variables. 
TABLEAU 2

Influence de la variété de luzerne sur la qualité nutritionnelle des protéines qui en sont extraites $(P X)$ pour la larve du ver de farine. Les résultats sont donnés sous la forme de la moyenne de 5 répétitions affectée de l'intervalle de confiance à $95 \%$. Deux moyennes suivies de la même lettre ne sont pas significativement différentes au seuil de $5 \%$ (test de Duncan).

Effect of lucerne variety on nutritional quality of the lucerne protein $(P X)$ for the yellow mealworm lurvae. Results are the average of 5 replicates. The mean value is given with confidence limits at the $95 \%$ level. Two means followed by the same letter are not significantly different at the $5 \%$ level (Duncan test).

\begin{tabular}{|c|c|c|c|c|c|c|}
\hline \multirow{3}{*}{$\begin{array}{c}\text { VARIÉTÉS } \\
\text { DE LUZERNE }\end{array}$} & \multicolumn{2}{|c|}{ TENEBRIO } & \multirow{2}{*}{\multicolumn{2}{|c|}{$\begin{array}{c}\text { TAUX DE SAPONINES } \\
\text { estimés par les tests } \\
\text { TRICHODERMA HÉMOLYSE } \\
\text { mg } / 100 \mathrm{~g} \text { de régime }\end{array}$}} & \multicolumn{2}{|c|}{ POUSSIN } \\
\hline & \multicolumn{2}{|c|}{$\begin{array}{l}\text { ASM mg/larve } \\
\end{array}$} & & & \multicolumn{2}{|c|}{ g/poussin } \\
\hline & $41,7(3,1) \mathrm{a}$ & $7,0(0,7) \mathrm{a}$ & 19,6 & 15,8 & 1086 & 591 \\
\hline LAHONTAN & $34,0(4,6) \mathrm{b}$ & $5,0(0,9) \mathrm{b}$ & 15,8 & 15,8 & 1099 & 595 \\
\hline ROBOT & $29,6(4,6) b c$ & $4,7(1,2) b c$ & 15,8 & 18,0 & 1125 & 609 \\
\hline DU PUITS & $28,4(2,4) b c$ & $4,1(0,5) b c$ & 20,2 & 22,8 & 1077 & 594 \\
\hline CAMPAGNE* & $24,8(3,7) c$ & $3,2(0,6) \mathrm{c}$ & 20,8 & 49,8 & 1083 & 587 \\
\hline VERNEUIL & $18,3(1,1) \mathrm{d}$ & $1,0(0,2) \mathrm{d}$ & 20,8 & 61,8 & 1018 & 548 \\
\hline RESIS & $14,6(1,7) \mathrm{e}$ & $0,6(0,3) \mathrm{de}$ & 21,5 & 54,8 & 1043 & 566 \\
\hline LUTÈCE & $8,8(1,4) \mathrm{f}$ & $0,2(0,3) \mathrm{e}$ & 22,1 & 61,2 & 1024 & 548 \\
\hline
\end{tabular}

Remarques :

* AU-PX-RURO correspond au mélange de 2 génotypes AU-PX et RURO.

* CAMPAGNE issu du mélange de 2 variétés EUROPE et VERNEUIL est assimilé à un PX standard.

\section{ANALYSE DE VARIANCE}

Variable étudiée : ASM

$\mathrm{F}=60$ avec $7 \mathrm{ddl}$ l'effet « variété » est très hautement significatif (F table $=2,36$ au seuil de $1 \%$ o $)$.

Variable étudiée : GPS

$F=77$ avec 7 ddl l'effet « variété " est très hautement significatif ( $F$ table $=2,36$ au seuil de $1 \%$ o).

\section{COEFFICIENTS DE CORRÉLATION ENTRE LES RÉSULTATS DES TESTS}

\begin{tabular}{|c|c|c|c|c|c|c|}
\hline & $\begin{array}{c}\text { TENEBRIO } \\
\text { ASM }\end{array}$ & $\begin{array}{c}\text { TENEBRIO } \\
\text { GPS }\end{array}$ & TRICHODERMA & HÉMOLYSE & $\begin{array}{c}\text { POUSSIN } \\
\text { QS }\end{array}$ & $\begin{array}{l}\text { POUSSIN } \\
\text { GP }\end{array}$ \\
\hline TENEBRIO ASM & 1 & & & & & \\
\hline TENEBRIO GPS & $0,98^{* * *}$ & 1 & & & & \\
\hline TRICHODERMA & $-0,64 \mathrm{NS}$ & $-0,65 \mathrm{NS}$ & 1 & & & \\
\hline HÉMOLYSE & $-0,89 * *$ & $-0,91 * *$ & $0,78 *$ & 1 & & \\
\hline POUSSIN QS & $0,77 *$ & $0,83 * *$ & $-0,82 *$ & $-0,86 * *$ & 1 & \\
\hline POUSSIN GP & $0,79 *$ & $0,84 * *$ & $-0,75 *$ & $-0,89 * *$ & $-0,98 * * *$ & 1 \\
\hline
\end{tabular}

Les critères de classement des variétés de luzerne, quantité d'aliment consommé et croissance pondérale des poussins, ont donné des résultats corrélés de façon significative à ceux obtenus avec le test biologique effectué sur les larves de $T$. molitor.

\section{L'effet d'un apport de cholestérol aux régimes expérimentaux}

L'addition de cholestérol aux aliments destinés aux larves de $T$. molitor a amélioré de façon significative les performances de ces insectes (tabl. 3), amélioration d'autant plus forte que la variété de luzerne est riche en saponines.

Cette amélioration, estimée par le calcul suivant :

ASM du régime - ASM du régime sans cholestérol

ASM du régime sans cholestérol

est représentée par le graphique 4 . Ce sont les variétés les plus riches en saponines qui sont les plus améliorées par l'addition de cholestérol.

\section{DISCUSSION ET CONCLUSION}

Les essais présentés ici montrent la forte corrélation qui existe entre les résultats des différentes méthodes
Figure 4

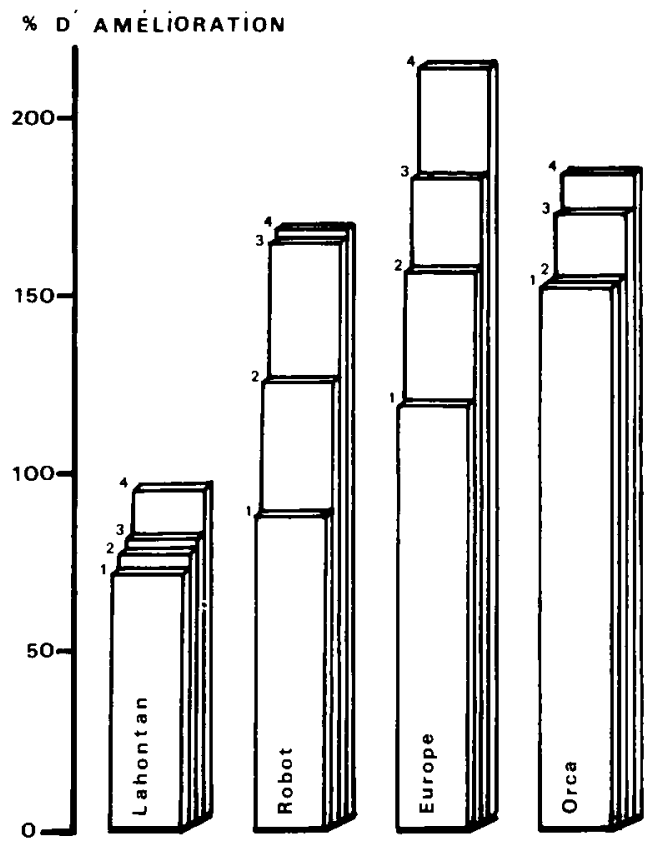

Amélioration de la quantité d'aliment métabolisé par Tenebrio molitor par augmentation des doses de cholestérol dans des régimes alimentaires contenant de la luzerne. Les 4 augmentations de 0,5 p. 100 sont notées de 1 à 4 sur la figure.

Increase of the dry food assimilated by Tenebrio molitor by increase of levels of cholesterol in diets prepared with lucerne. Four $0.5 \%$ increases are noted 1 to 4 in this graph. 
TABLEAU 3

Effets, sur les larves du ver de farine, de l'incorporation de doses croissantes de cholestérol dans des régimes alimentaires à base de feuilles de luzerne de 4 variétés différemment pourvues en saponines.

Les résultats sont donnés sous la forme de la moyenne de 5 répétitions affectée de l'intervalle de confiance à $95 \%$.

Effects on yellow mealworm larvae of increase of cholesterol in diets which contained leaves of 4 varieties of lucerne. Results are the average of 5 replicates. The mean value is given with confidence limits at the $95 \%$ level.

\begin{tabular}{|c|c|c|c|c|}
\hline VARIÉTÉS & LAHONTAN & ROBOT & EUROPE & ORCA \\
\hline CHOLESTÉROL (\%) & ASM mg/l & ASM mg/l & ASM mg/l & ASM mg/l \\
\hline $\begin{array}{l}0 \\
0,5 \\
1 \\
1,5 \\
2\end{array}$ & $\begin{array}{r}60,8(3,1) \\
104,5(9,8) \\
108,3(8,7) \\
110,7(9,2) \\
119,0(9,9)\end{array}$ & $\begin{array}{l}34,3(5,1) \\
64,6(5,7) \\
77,6(2,5) \\
90,5(6,0) \\
92,0(9,9)\end{array}$ & $\begin{array}{l}20,9(1,2) \\
45,7(5,7) \\
53,8(1,8) \\
59,1(5,2) \\
65,7(2,8)\end{array}$ & $\begin{array}{l}18,4(1,5) \\
46,5(1,6) \\
46,7(2,2) \\
50,2(5,8) \\
52,3(2,0)\end{array}$ \\
\hline CHOLESTÉROL $(\%)$ & GPS mg/1 & GPS mg/l & GPS mg/l & GPS mg/l \\
\hline $\begin{array}{l}0 \\
0,5 \\
1 \\
1,5 \\
2\end{array}$ & $\begin{array}{r}5,1(1,7) \\
13,1(1,8) \\
13,9(1,7) \\
14,9(0,8) \\
16,0(1,0)\end{array}$ & $\begin{array}{r}2,3(1,1) \\
6,8(1,8) \\
9,4(0,5) \\
11,9(2,0) \\
11,5(2,7)\end{array}$ & $\begin{array}{l}0,3(0,1) \\
4,7(1,3) \\
5,5(0,2) \\
6,4(1,5) \\
7,1(1,2)\end{array}$ & $\begin{array}{l}0,7(0,5) \\
4,6(0,2) \\
4,8(1,3) \\
5,2(1,0) \\
5,6(0,8)\end{array}$ \\
\hline \multicolumn{5}{|c|}{ TABLEAUX D'ANALYSE DE VARIANCE } \\
\hline SOURCE DE VARIATION & SCE & ddl & $\mathrm{CM}$ & $\mathrm{F}$ \\
\hline $\begin{array}{l}\text { Variété } \\
\text { Dose de cholestérol } \\
\text { Répétition } \\
\text { Interaction Variété X Dose }\end{array}$ & $\begin{array}{r}51400 \\
29300 \\
285 \\
1950\end{array}$ & $\begin{array}{r}3 \\
4 \\
4 \\
12\end{array}$ & $\begin{array}{r}17100 \\
7320 \\
71 \\
163\end{array}$ & $\begin{array}{c}657^{* * * *} \\
281 * * * \\
3 * \\
6^{* *}\end{array}$ \\
\hline $\begin{array}{l}\text { Erreur } \\
\text { Totale }\end{array}$ & $\begin{array}{r}1980 \\
84900\end{array}$ & $\begin{array}{l}76 \\
99\end{array}$ & 26 & \\
\hline \multicolumn{5}{|l|}{2 - Etude de la variable GPS } \\
\hline SOURCE DE VARIATION & SCE & ddl & $\mathrm{CM}$ & F \\
\hline $\begin{array}{l}\text { Variété } \\
\text { Dose de cholestérol } \\
\text { Répétition } \\
\text { Interaction Variété X Dose }\end{array}$ & $\begin{array}{r}1120 \\
805 \\
6 \\
84\end{array}$ & $\begin{array}{r}3 \\
4 \\
4 \\
12\end{array}$ & $\begin{array}{c}375 \\
201 \\
1,5 \\
7\end{array}$ & $\begin{array}{c}455^{* * *} \\
244 * * * \\
1,8 \mathrm{NS} \\
8,5 * *\end{array}$ \\
\hline $\begin{array}{l}\text { Erreur } \\
\text { Totale }\end{array}$ & $\begin{array}{r}63 \\
2080\end{array}$ & $\begin{array}{l}76 \\
99\end{array}$ & 0,8 & \\
\hline \multicolumn{5}{|c|}{ AMÉLIORATION DE ASM $(\%)$} \\
\hline VARIETTES & LAHONTAN & ROBOT & EUROPE & ORCA \\
\hline \multicolumn{5}{|c|}{ RÉFÉRENCES CHOLESTÉROL } \\
\hline 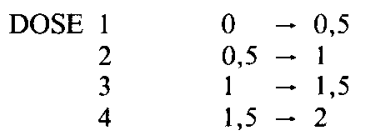 & $\begin{array}{l}71,9 \\
78,1 \\
82,1 \\
95,7\end{array}$ & $\begin{array}{r}88,3 \\
126,2 \\
163,8 \\
168,2\end{array}$ & $\begin{array}{l}118,7 \\
157,4 \\
182,8 \\
214,3\end{array}$ & $\begin{array}{l}152,7 \\
153,8 \\
172,8 \\
183,7\end{array}$ \\
\hline
\end{tabular}

de dosage biologique des saponines de la luzerne. Le test Tenebrio ayant été décrit par ailleurs comme une méthode simple à mettre en œuvre, peu onéreuse et ne nécessitant que très peu de matière première (PRACROS, 1982), son utilisation peut être envisagée au même titre que le test Trichoderma (réalisé en Italie) et le test hémolyse (appliqué en Hongrie).

Ces travaux, menés simultanément par plusieurs équipes d'un groupe de travail, montrent clairement l'effet antinutritionnel des saponines pour les poussins, effet qui justifie la mise en place d'un programme de sélection pour l'obtention de variétés sans saponines.

Chaque année, le sélectionneur dispose d'un grand nombre de lignées parmi lesquelles il faut choisir : le test Tenebrio peut être un bon « outil de sélection » au même titre que les autres essais biologiques utilisables actuellement.

\section{Reçu le 16 septembre 1987. Accepté le 5 décembre 1987.}

\section{REMERCIEMENTS}

Nous remercions tous les membres du groupe de travail « saponines/luzerne " qui ont bien voulu nous autoriser à reproduire les résultats des tests qu'ils ont effectués :

W. Pietracsek, I.N.R.A., Station d'amélioration des plantes, 86600, Lusignan.

I. Bocsa, Gate, Institut de Recherches Agronomiques, 3356 Kompolt, Hongrie.

L. LaCassagne, I.N.R.A., Station de Recherches Avicoles, Centre de Tours-Nouzilly, 37380 Monnaie. 


\section{RÉFÉRENCES BIBLIOGRAPHIQUES}

Applebaum S. W., Birk Y., 1979. Saponins, 539-566. In G. A. RosentHal et D. H. JANZEN " Herbivores, their interaction with secondary plants metabolites", Acad. Press, N. Y., 718 p.

Birk Y., Péri I., 1980. Saponins, 161-180. In I. E. Liener « Toxic constituents of plant foodstuffs ", Acad. Press, N. Y., $502 \mathrm{p}$.

Cheeke P. R., 1971. Nutritional and physiological implication of saponins : a review. Can. J. anim. Sci., 51, 621-632.

Cheeke P. R., 1976. Nutritional and physiological properties of saponins. Nutr. Rep. int., 13, 316-324.

Fraenkel G., Blewetl M., Coles M., 1950. The mutrition of the mealworm Tenebrio molitor L. (Tenebrionidae, Coleoptera). Physiol. zool., 23, 92-108.

Gestetner B., 1971. Structure of saponin from lucerne Medicago sativa. Phytochem., 10, 2221-2223.

Gestetner B., Assa Y.. Hénis Y., Tencer Y., Rotman M.. Birk Y.. Bondi A., 1972. Interaction of lucerne saponins with sterols. Biochim. Biophys. Acta, 270, 181-187.

Horber E., Leath K. T., Berrang B., Marcarian V., Hanson C. H., 1974. Biological activities of saponins components from Du Puits and Lahontan alfalfa. Ent. exp. appl., 17, 175-185.

Ishaaya I., Birk Y., Bondi A., Tencer Y., 1969. Soybeans saponins. IX. Studies of their effect on birds, mammals and cold-blooded organisms. J. Sci. Food Agric., 20, 433-436.
Leath K. T., Davis K. H., Wall M. E., Hanson C. H., 1972. Vegetative growth responses of alfalfa pathogens to saponins and other extracts from alfalfa (Medicago sativa). Crop. Sci., 12, 851-856.

Pedersen N. W., Wang L. C., 1971. Modification of saponin content of alfalfa through selection. Crop. Sci., 11, 833-835.

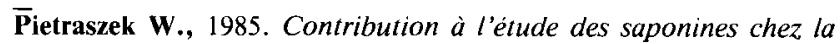
luzerne (Medicago sativa L.): rôle antinutritionnel, variabilité génétique, aspects technologiques. Thèse, Université de Paris-Sud, Centre d'Orsay, 68 p.

Pracros P., 1982. Intérêt comparé de l'estimation biologique de la valeur nutritionnelle des sources protéiques par des vertébrés (rats et poulets) et par un insecte (Tenebrio molitor). C. R. Acal. Agric., 1279-1285.

Pracros P., 1984. Influence de quelques substances allelochimiques sur la physiologie alimentaire des larves du ver de farine, Tenebrio molitor L. Bull. OILB/SROP, 7, 4, 65-66.

Ritter F. J., Wientjens W. H. J. M., 1967. Sterol metabolism of insects. T.N.O. Nieuws, 381-392.

Shany S., Birk Y., Gestetner B., Bondi A., 1980. Preparation, characterisation and some properties of saponins from lucerne tops and roots. J. Sci. Food Agric., 21, 508-510. 